\title{
Energy efficient intelligent routing in WSN using dominant genetic algorithm
}

\author{
Shanthi D L ${ }^{1}$, Dr. Keshava Prasanna ${ }^{2}$ \\ ${ }^{1}$ Department of Information Science and Engineering, BMSIT \& M, Bangalore, India \\ ${ }^{2}$ Department of Computer Science and Engineering, CIT, Tumkur, India
}

\begin{tabular}{l}
\hline Article Info \\
\hline Article history: \\
Received Feb 14, 2019 \\
Revised Aug 19, 2019 \\
Accepted Aug 30, 2019 \\
\hline
\end{tabular}

\section{Keywords:}

Energy efficient

Genetic algorithm

Heuristic

Mobility

Network lifetime

\begin{abstract}
In the current era of wireless sensor network development, among the various challenging issues, the life enhancement has obtained the prime interest. Reason is clear and straight: the battery operated sensors do have limited period of life hence to keep the network active as much as possible, life of network should be larger. To enhance the life of the network, at different level different approaches has been applied, broadly defining the proper scheduling of sensors and defining the energy efficient communication. In this paper heuristic based energy efficient communication approch has applied. A new development in the Genetic algorithm has presented and called as Dominant Genetic algorithm to determine the optimum energy efficient routing path between sensor nodes and to define the optimal energy efficient trajectory for mobile data gathering node. Dominancy of high fitness solution has included in the Genetic algorithm because of its natural existence. The proposed solution has applied the connection oriented crossover and mutation operator to maintain the feasibility of generated solution. The proposed solution has applied with various simulation experiments under two different scenarios: in first case energy efficient routes among the sensors have explored to deliver the information from source sensor to the sink node and in second case, energy efficient route among all local data hubs for mobile data gathering node has obtained. The proposed solution performances have been analyzed quantitatively and analytically. It has observed with various experimental results that proposed method not only has delivered the better solution but also has faster convergence and high level of reliability in compared to conventional form of Genetic algorithm.
\end{abstract}

Copyright (c) 2020 Institute of Advanced Engineering and Science. All rights reserved.

\section{Corresponding Author:}

Shanthi D L,

Department of ISE,

BMS Institute of Technology, Bangalore-64, India.

Tel: +91-9449176450

Email: gopalaiahshanthi@bmsit.in

\section{INTRODUCTION}

The innovations and progress in wireless communication and micro-sensing delivers a useful means to observe environment, ecological system, personal health, in building smart-homes, military surveillance, vehicle monitoring and so on. Any sensor network is implemented using a huge number of tiny devices that are limited by sensing, processing, transmitting abilities, and are battery powered. Collecting data is the most common and essential tasks in sensor networks; effectiveness of executing data collection operation defines network lifetime. Due to the inadequate radio resources and the energy limitation on each sensor node, it is very motivating task to extend the network lifetime while maintaining certain data collection rate. Several ways are used to transfer the sensed data from sensor nodes to the base station for processing say multi-hop. Energy is the primary apprehension to Wireless Sensor Networks (WSNs) for all its transmis- 
sion and reception of data but, consumes a lot of energy to synchronize and to ensure the location awareness of the sensors.

A number of researchers have suggested mobility as a solution to the problem of data gathering [1-7]. Contemporary study has revealed that a significant decrease in communication energy consumption using controlled mobility in WSN. Example, a Mobile Base Station (MBS) can go from place to place in the sensing area to collect data from nodes using short-range communications. Introduction of mobile nodes in to WSN, energy utilization of static nodes may be reduced. Unscheduled mobility of mobile node might increase latency in data collection. Hence, it is necessary that trajectory obtained by MBS should be optimal in the sense of travel distance and invested energy. Major limiting factor of a WSN is its node's energy, which demands the design of an energy-efficient routing protocol that increases the whole system performance.

In this paper two different perspectives of energy saving scheme in WSN has presented. In the first case the need of routing path between two sensors obtained which carry the minimum expenditure of energy while in other case the trajectory of mobile data gathering has been defined to collect the data from different local base nodes which already has the information of all sensors belonging to their clusters. It is assumed that in the first case there is no mobility observed among the sensors while in the second case cluster heads were already available.

Because of complexity involved with the problem is NP hard, heuristic approach has applied to achieve the solution. Among the various possibilities, the heuristic approach of Genetic algorithm has proved with the time as one of the more dominant and wide applicable method. There are various issues with conventional approach of Genetic algorithm (CGA) like unfair selection of parents to produce offspring, locus of crossover point, strategy of mutation applied and more importantly balancing between exploration against exploitation. To overcome these issues, a dominant form of Genetic algorithm has proposed which provide fair opportunities to each and every parent in participation of offspring creation helps in exploration, while elite members of the population deliver the rule of dominancy to exploit better one. A fair selection process through tournament selection has applied which deliver the number of opportunities to each and every one to prove itself rather than fitness oriented selection which causes faster convergence with sub-optimal solution. From simulation experiments it has been observed that there is better and faster routing strategy achieved within very less number of iterations which makes the proposed solution very computation efficient also.

\section{RELATED WORK}

In the direction of energy saving scheme in WSN, there were number of different approaches by different researchers have been proposed in the past. In [1], using the theory of potential in physics, an EnergyBalanced Routing Protocol (EBRP) had been designed by creating a mixed virtual potential field in terms of depth, energy density, and residual energy. In [2], the nodes deployed in WSN uses the renewable energy generated by solar panels built over for routing and sensing, here energy-efficient routing protocol for energy harvesting WSN is designed and implemented. While choosing route the parameters considered are transmission quality, energy depletion and energy wasting and the effect of bit error rate (BER). To maximize this WSN feature, the data and message delivery routes are carefully chosen so that overall energy consumption is minimized. M. Bayani [3] had analyzed a detailed comparison between typical WSN protocols and their impacts over the WSN lifetime and perceived that flat and cluster-based protocols can increase WSN lifetime in different ways. Study has presented in [4] over basic optimization of base-station positioning in WSN so that the data from the sensors can be communicated in an energy-efficient manner. Chaonan Wang [5] had proposed a prototypical and evaluated the consistency and lifetime of a sensor node in three typical set-ups, providing precise reliability analysis of WSN systems. Substantial improvement in WSN lifetime could be attained by introducing standby or spare nodes. These spare nodes are substituted, once any prime (original) node is depleted with energy. Bilal AbuBakr [6] had proposed the LEACH-SM protocol, this is a modified form of Low-Energy Adaptive Clustering Hierarchy (LEACH) designed by providing an optimum spare nodes and energy management in spares. Mariam Akbar [7] had proposed Balanced Energy-Efficient Network Integrated Super Heterogeneous for heterogeneous WSNs to improve stability, lifetime and throughput. In the direction of improving the performance of WSN with respect to energy requisite and to increase network lifetime a communication/computation energy trade-off need to be analyzed [8]. The analysis could be made at network-level (i.e., all nodes in the network use the same strategy) or at a node-level (i.e., sensor nodes do not necessarily have identical strategies). Robert M.Curry [9] had explored a number of research methodolo- 
gies and leeway to the problem that includes online routing, clustering methods, and lifetime maximization on specially structured network. Based on Lagrange relaxation method, [12] had proposed an energy optimization method to assure delay constraint. An objective function has been proposed in terms of energy consumption and delay and also defined a method to find an optimal multiplier for that objective function. Based on Ant colony algorithm optimal path for routing has proposed in [13]. Jian Shen [14] had proposed an energy-efficient centroid-based routing protocol (EECRP) for WSN assisted IoT to get better performance of the network. Rumor routing is another typical random walk routing protocol defined, but the problem, is not scalable and can lead to spiral paths. Hsiang-Hung Liu [15] had considered straight-line routing (SLR) to decrease the energy utilization of sensor nodes in WSNs. Locality of sink node may considerably affect the energy dissipation and throughput of the network. Yahya Kord Tamandani [16] had given investigation over an optimum position for the sink node in such a way that the sum of distances from all the sensor nodes to the sink node is minimized. An Energy Efficient Connected Coverage (EECC) scheduling is made use to extend the lifetime of the WSN is given in [17]. Secure and energy-efficient method of optimization has been proposed in [18] using the Dij-Huff Method. Turki A. Alghamdi [19] had proposed a WSN-based multi-hop network infrastructure, to increase network lifetime by optimizing the routing strategy. A proposal of a novel routing architecture has suggested in [20] for severe environment monitoring in heterogeneous WSN. The aim was to improve the stability period and network lifetime by restriction the distance between the sensor nodes and the gateway node by mitigating the hot-spot problem in the network. Random projection based on compressed sensing might decrease the volume of data communicated in a WSN, and efficient routing could ease the network traffic. Jianhua Qiao [21] presented a Random projection-Polar coordinate-Chain routing (RPC) scheme to develop the time and energy efficient protocol. The review in [22] had presented the state of the art in the energy management schemes, and the available challenges in the area of WSN. [23] had proposed a method to reduce the energy consumption by energy balancing in clusters among all sensor nodes to minimize the energy dissipation during network communications. Arun L.Kakhandki [24] presented a distributed MAC and transceiver optimization technique for selective hop device selection to minimize energy consumption per bit and maximize the lifetime of sensor network.

The study in [25] presented a survey approach for different aspects involved with Heterogeneous Wireless Sensor network and design issues for routing in heterogeneous environment. Hemavathi P [26] had applied the modified version of Bacteria Foraging Optimization to optimize the energy consumption in data aggregation process in WSN. Basavaraj G.N [27] had applied Low Latency and Energy Efficient Routing (LLEER) design for heterogeneous WSN to provide the trade-off between energy efficiency and latency requirement. Chaitra HV [28] had presented cluster head selection based life enhancement of network using a Multi-objective imperialist competitive algorithm (MOICA) .

\section{PROPOSED WORK}

\subsection{Mathematical modeling of problem}

Mathematical representation of the objective function can be defined as the minimization of the total energy spends over the considered route path. In graphical model of simulated network of WSN G (V, E), Energy efficient routing between defined sensor nodes $(\mathrm{P}, \mathrm{Q})$ can be consider as finding a number of possible paths $\left\{P_{i} \mid i \in\{0,1, \ldots\}\right\}$ sequentially over a set of graphs $\left\{G_{i} \mid i \in\{0,1, \ldots\}\right\}$, which must carry the minimum energy cost path as given in equation (1)

$$
C F(P Q)=\operatorname{Min}\left\{\sum_{l \in P_{i}(s, r)} C F_{l}\right\}
$$

The optimal routing has transformed as a problem of optimization where objective was to minimize the total invested travel cost as a function of energy. The optimization of objective function has achieved by applying a modified form of Genetic algorithm which has used a more natural mechanism of "Dominancy" in the formation of new solution population. Genetic algorithm has shown great interest by a number of researchers $[10,11]$

\subsection{Dominant genetic algorithm}

A solution population is initialized for a defined source-destination sensor pair which uses a concept of connected possible solution in the feasibility domain of possible paths. An equal opportunity 
for each parent is performed through uniform distribution probability in the process of offspring formation. This step is very natural and provides the facility of better exploration of the solution domain. One point crossover operator has applied which carried the node connected feature, where a set of common nodes in both parents form the possible locus of crossover point. If there were more than one possible locus in the set, a uniform random process has applied to select the particular one. Such kind of cross over maintain the explored solution under the feasibility domain and may cause the change in the length of generated offspring. A dynamic mutation strategy for each locus of offspring has under low mutation probability to maintain the diversity is used. A possible candidate of mutation is the member from a set of all possible connected sensor nodes from the just previous sensor. Again such process maintains the feasibility of explored solution. Once the number of generated offspring's is same as parent population size, both populations are merged to form a selection pool where a tournament selection process is applied. In this selection process for each member a number of opponent members are selected randomly and it depends upon the fitness comparison and a tournament score declared. The higher scored members form a new population. In the new population, under a defined range, a random number of members are selected as elite members as well as poorest members depending upon their fitness. Elite member generate the offsprings and they replace the poorest member from the population if their fitness is better. Such kind of dominancy is very obvious and could be observed in human population as well. This dominancy involvement makes population more fitter as well as increase the rate of convegence without compromise with the exploration level. The concept of conditional niche has also applied where the previous best observed solution replaces the weakest member of the solution if the fitness allowed. The obtained final population has considered as the next generation population for further process. The functional block diagram of proposed algorithm has shown in Figure 1.The number associated with the edges indicate the flow of process sequentially.

\subsubsection{Chromosome representation}

Initial solution population has defined under a constraint based definition of nodes connection between a predefined source-destination pair. The following steps are used in the formation of each member in the initial population.

\section{Pseudo code for initial solution formation:}

1. Initialization of Population size

For each member: fixed Sr \& Dt: start solution as $\mathrm{S} \longleftarrow$ [Sr]

2. Find Possible neighbors of last member of $\mathrm{S}$, say $\mathrm{NR} \longleftarrow\{\mathrm{Ni}, \mathrm{Nj}, \ldots . \mathrm{Nm}\}$

3. Add a node from set $\mathrm{NR}$ to $\mathrm{S}:[\mathrm{Sr}, \mathrm{Nj}] \leftarrow$ [NR ] $\longleftarrow$ [Uniform Random Process]

4. Go to step 2 if the last node of [S(end)] \# Dt.

\subsubsection{Connectivity based crossover operator and mutation}

In the considered form of WSN, normal course of crossover operation is not possible because of limited connectivity association with other available sensor nodes. As in the case of normal process of crossover, any random position is considered as point of crossover, obtained offspring may carry the infeasibility and it's not possible to recorrect that infeasibility with penalty process. In the proposed form of crossover same locus position will not appear, instead of that same node will be considered as the position of crossover as shown in Figure 2. As in Figure 2 (a), parents P1 AND P2 have taken for crossover. There are two attractors available in chromosomes (N3, N2) and (N2, N6), where the first position (like N3) is the position from first parent and a second position (N2) is the position from second parent. Other positions are not allowed to crossover; because they will make the solution unfeasible. It is also observed that crossover can cause the change in chromosome length. In this paper, possible domain of change under mutation with each node is the possible number of nodes, which are connected with their neighbors only.

In this paper, possible domain of change under mutation with each node is the possible number of nodes, which are connected with their neighbor's only. For a node which has to mutated first all the connected nodes have explored in the sensor network and nodes which already existed beside in the solution, discarded. From the remaining nodes, through uniform random selection process a node has selected as the mutated node. For example the mutation strategy for the node 9th of the offspring $\mathrm{O} 2$ has shown in Figure 2(c). First for the node 9, all the connected nodes have explored in the sensor network and it has appeared that nodes 1, 8, 19, 17 and 4 are the connected nodes. Nodes 1 and 8 which were already existed beside in the solution discarded 
and among remaining nodes 4, 17 and 19, a node (for example node 19 in MO2) has selected through uniform random selection process.

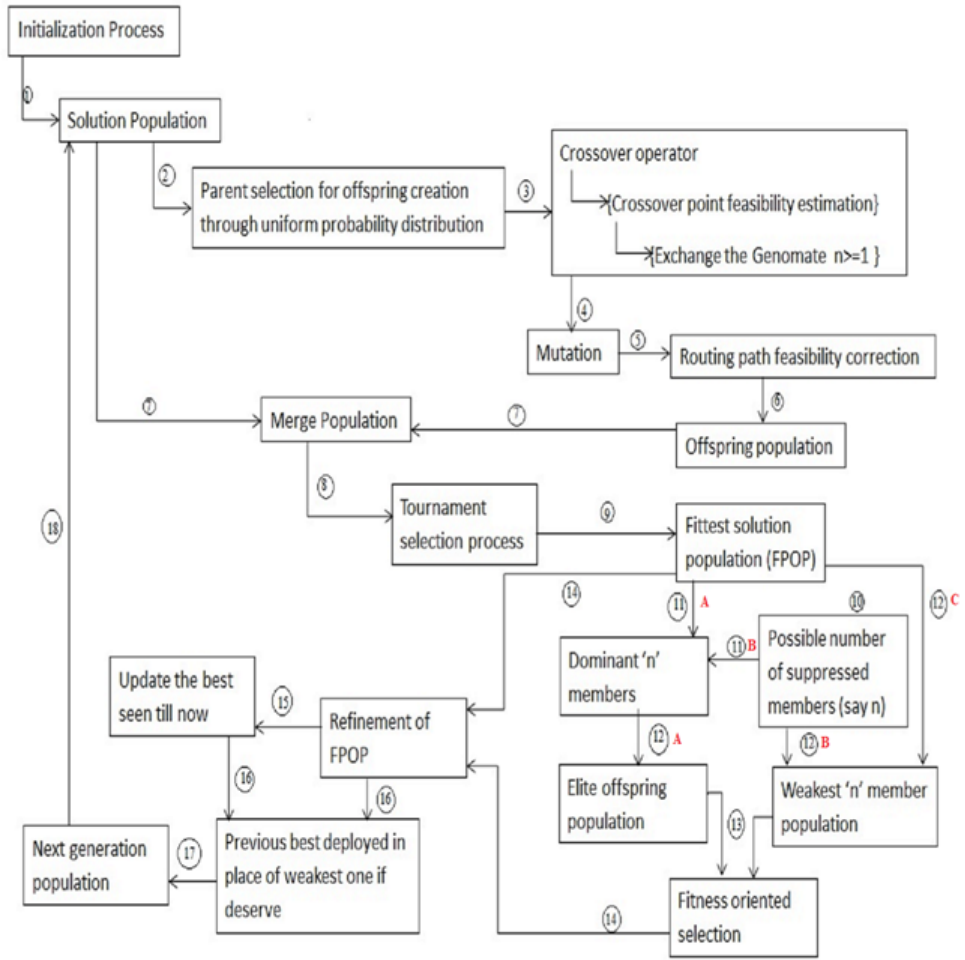

Figure 1. Functional representation of proposed Dominant Genetic algorithm

\begin{tabular}{|l|l|l|l|l|l|l|l|l|l|}
\hline & Source & N1 & N2 & N3 & N4 & N5 & N6 & Destination & Chrom.Length \\
\hline P1 & 1 & 6 & 5 & 8 & 7 & 13 & 11 & 15 & 8 \\
\hline P2 & 1 & 9 & 8 & 10 & 2 & 12 & 5 & 15 & 8 \\
\hline
\end{tabular}

(a)

\begin{tabular}{|l|l|l|l|l|l|l|l|l|l|l|}
\hline & & & & & & & & & & Chrom.Length \\
\hline O1 & 1 & 6 & 5 & 8 & 10 & 2 & 12 & 5 & 15 & 9 \\
\hline O2 & 1 & 9 & 8 & 7 & 13 & 11 & 15 & & & 7 \\
\hline
\end{tabular}

(b)

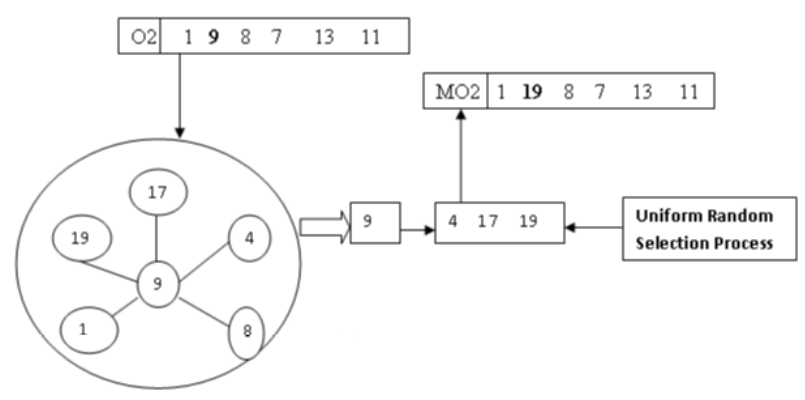

(c)

Figure 2. Connectivity based crossover, (a) parents selected for crossover (b) generated offspring after crossover (c) Mutation Strategy 


\subsection{Advantages of proposed solution}

The applied form of proposed solution is having numerous advantages in terms of finding the efficient solution.

1. Dynamic length of solution in DGA has provided the possibilities of high level of exploration as well as computational ease.

2. Connected nodes cross-over operation only explore new solution and never destroy the developed solution (while in conventional Genetic algorithm crossover operator cause of construction as well as destruction also which may cause of more number of iterations to appear same solution or may not deliver the optimal solution at all).

3. Connected node mutation strategy causes of new solution exploration with minimal computational cost.

4. Routing path feasibility correction process causes of another process to increase the level of diversity in the solution.

5. Chance of every parent equally in offspring creation cause of deeper exploration in solution space.

6. Dominant process provides high level of balancing between exploration vs exploitation. Explored Elite offsprings exploited immediately by suppressing the weakest members and cause of faster convergence.

\section{SIMULATION RESULT}

Two different possibilities of routing scenarios have explored. In the first case number of sensors form a network and communication takes place between the source node and sink node, as shown in Figure 3 simulated network 1 . Such a scenario needs an optimal path so that minimum energy is invested over the communication. In second case, data gathering node moves from centroid of one cluster to another cluster to collect the data and after visiting all the clusters it will return back to the starting position, as shown in Figure 7 simulated network 2. Both cases can be handled as problem of path optimization.

\subsection{Case1. network1}

In the area of 200 X 200 square units simulation experiment has done in MATLAB environment by random placement of sensors through the uniform distribution. A Total of 50 sensors have deployed and with the communication range of 50 units, connectivity in the network among the sensors has formed. Rather than considering the direct physical Euclidean distance between the connected sensors, a uniform random number in the range of $[10,20]$ has selected to model invested energy with the irregularities existed in the practical geographical environment. A population size of 20 has selected with crossover rate as 1 if feasibility exist otherwise equal to 0 . A low probability of mutation rate 0.1 has considered in inheriting the parent quality. For a given node pair, the process has iterated upto to 20 iterations and 10 independent trials to estimate the statistical significance. Performance qualities have measured in terms of cost of objective function value as well as time of solution stability. Figure 4 shows large change of convergence in CGA under 10 independent trails and Figure 5 shows a tight convergence in DGA under 10 independent trails for network 1. Figure 6 shows a mean convergence for CGA and DGA for 10 independent trails. Statistically an improvement in the performance is seen in DGA compared to CGA from Table 1 and Table 2 respectively.

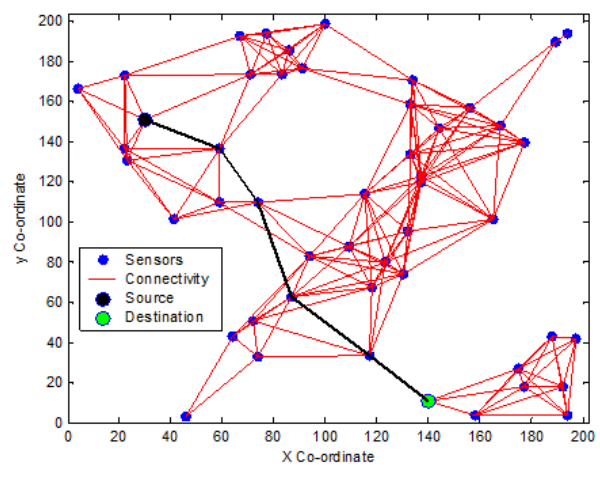

Figure 3. Simulated network 1 with obtained route between nodes

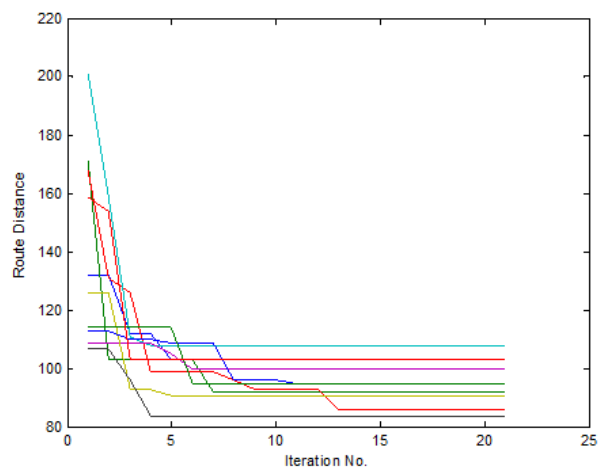

Figure 4. Convergence in CGA under 10 independent trials 


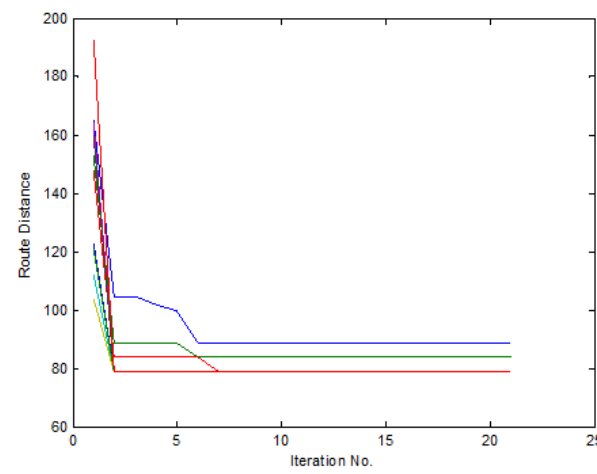

Figure 5. Convergence in DGA under 10 independent trials

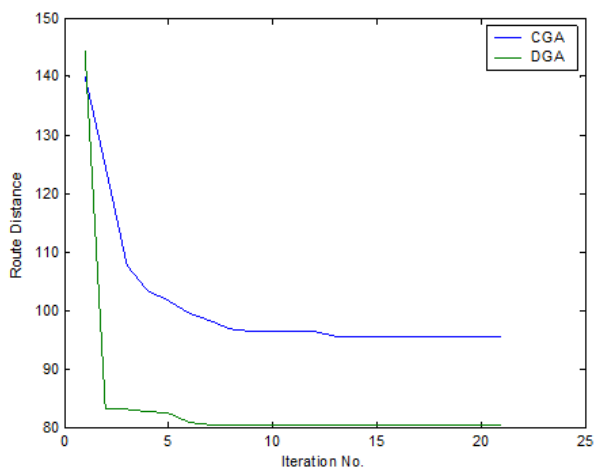

Figure 6. Mean Convergence in CGA and DGA over 10 independent trials

Table 1. Performance of CGA and DGA under 10 independent trials

\begin{tabular}{ccccc}
\hline Trial No. & \multicolumn{2}{c}{ CGA } & \multicolumn{2}{c}{ DGA } \\
$\mathrm{P}=20 ; \mathrm{S}=50 ; \mathrm{C}=50$ & Route Cost & Iteration Cost & Route Cost & Iteration Cost \\
\hline 1 & 103 & 5 & 89 & 6 \\
2 & 92 & 7 & 84 & 6 \\
3 & 86 & 13 & 79 & 7 \\
4 & 108 & 4 & 79 & 2 \\
5 & 100 & 6 & 79 & 2 \\
6 & 91 & 5 & 79 & 2 \\
7 & 84 & 4 & 79 & 2 \\
8 & 95 & 11 & 79 & 2 \\
9 & 95 & 6 & 79 & 2 \\
10 & 103 & 3 & 79 & 2 \\
\hline
\end{tabular}

Table 2. Statistical behavior of performance in CGA and DGA

\begin{tabular}{ccccc}
\hline & \multicolumn{2}{c}{ CGA } & \multicolumn{2}{c}{ DGA } \\
& Route Cost & Iteration Cost & Route Cost & Iteration Cost \\
\hline Best & 84.0 & 3.0 & 79.0 & 2.0 \\
Worst & 108.0 & 13.0 & 89.0 & 7.0 \\
Mean & 95.7 & 6.4 & 80.5 & 3.3 \\
Std.Dev. & 7.78 & 3.2 & 3.4 & 2.1 \\
\hline
\end{tabular}

\subsection{Case1. network2}

A network with different source and sink node set up is considered to test the performacne of DGA over CGA for 10 independent trails is shown in Figure 7. It has observed with Network 1 and Network 2, a very sharp benefit is obtained with DGA compared to CGA. Figure 8 shows a large change in the covergence characteristics with CGA while Figure 9 with DGA follow a very tight relation among the different trials. The clear difference of convergence between CGA and DGA have shown in Figure 10 for netowrk2 over 10 independent trails. The statistical analysis from Table 3 shows that DGA has remarkably faster and lower route path cost. Table 4 shows statistical behavior of performance in CGA and DGA for best, worst and mean cases approximately 50\% increase. In both cases DGA has delivered the lower value of route cost as well as strong reliability in performance. The computation cost is about 2 to 3 iterations in DGA while CGA has taken around 7 iterations. 


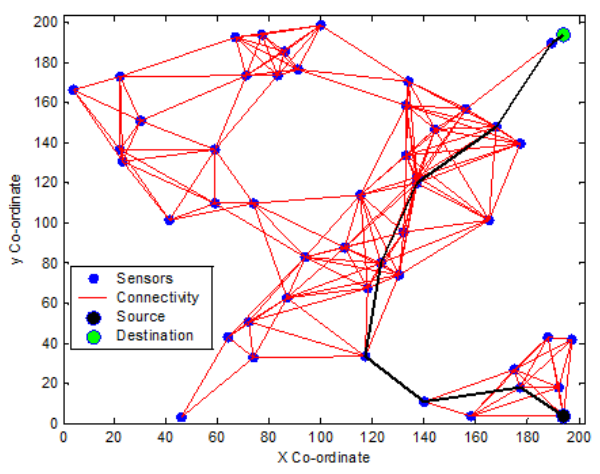

Figure 7. Simulated network 2 with obtained route between nodes

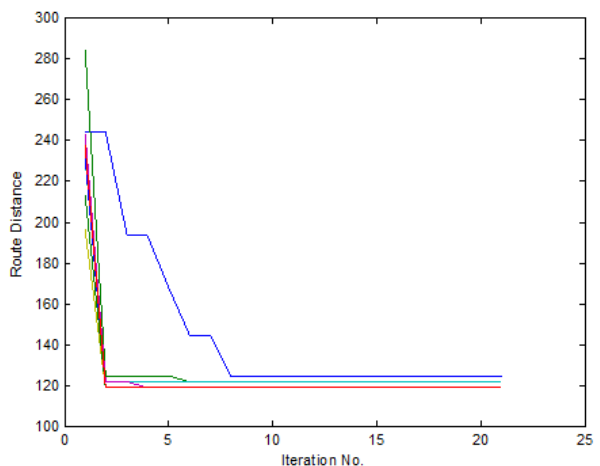

Figure 9. Convergence in DGA under 10 independent trials

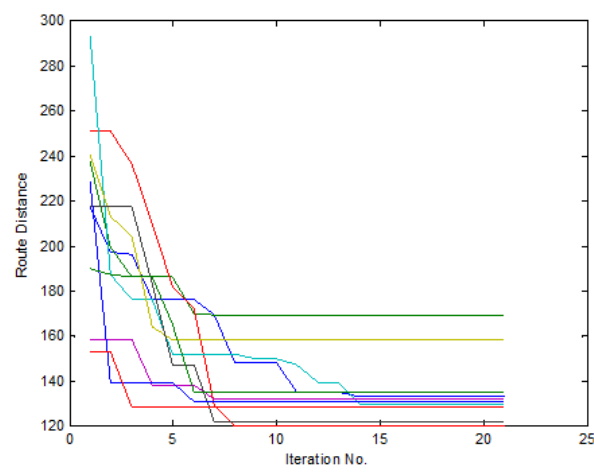

Figure 8. Convergence in CGA under 10 independent trials

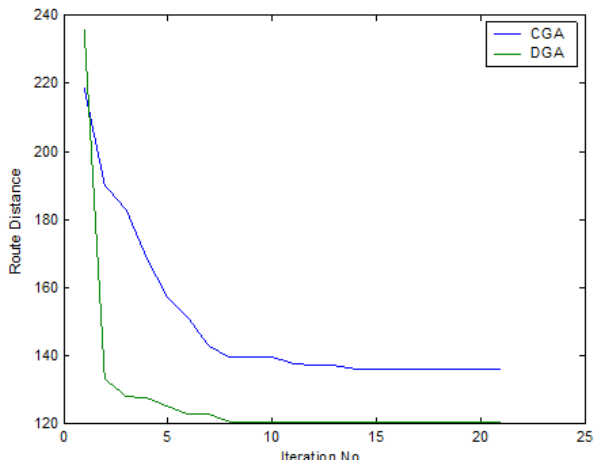

Figure 10. Mean Convergence in CGA and DGA over 10 independent trials

Table 3. Performance of CGA and DGA under 10 independent trials

\begin{tabular}{ccccc}
\hline Trial No. & \multicolumn{2}{c}{ CGA } & \multicolumn{2}{c}{ DGA } \\
$\mathrm{P}=20 ; \mathrm{S}=50 ; \mathrm{C}=50$ & Route Cost & Iteration Cost & Route Cost & Iteration Cost \\
\hline 1 & 133 & 14 & 125 & 8 \\
2 & 169 & 7 & 122 & 6 \\
3 & 120 & 8 & 122 & 2 \\
4 & 130 & 14 & 122 & 2 \\
5 & 132 & 7 & 119 & 4 \\
6 & 158 & 5 & 119 & 2 \\
7 & 122 & 7 & 119 & 2 \\
8 & 131 & 6 & 119 & 2 \\
9 & 135 & 6 & 119 & 2 \\
10 & 128 & 3 & 119 & 2 \\
\hline
\end{tabular}

Table 4. Statistical behavior of performance in CGA and DGA

\begin{tabular}{ccccc}
\hline & \multicolumn{2}{c}{ CGA } & \multicolumn{2}{c}{ DGA } \\
& Route Cost & Iteration Cost & Route Cost & Iteration Cost \\
\hline Best & 120.0 & 3.0 & 119.0 & 2.0 \\
Worst & 169.0 & 14.0 & 125.0 & 8.0 \\
Mean & 135.8 & 7.7 & 120.5 & 3.2 \\
Std.Dev. & 15.5 & 3.6 & 2.1 & 2.5 \\
\hline
\end{tabular}




\subsection{Trajectory of data gathering node}

A simple model of mobile data gathering in WSN has shown in Figure 11. Here each cluster is having a cluster head which has all the information of cluster center. Rather than getting the information from each sensor, information collection by data gathering node can save lot of energy, and also the delay in information transmission can completely depend upon the trajectory quality. Hence it is necessary; the selected trajectory should be optimal and faster.

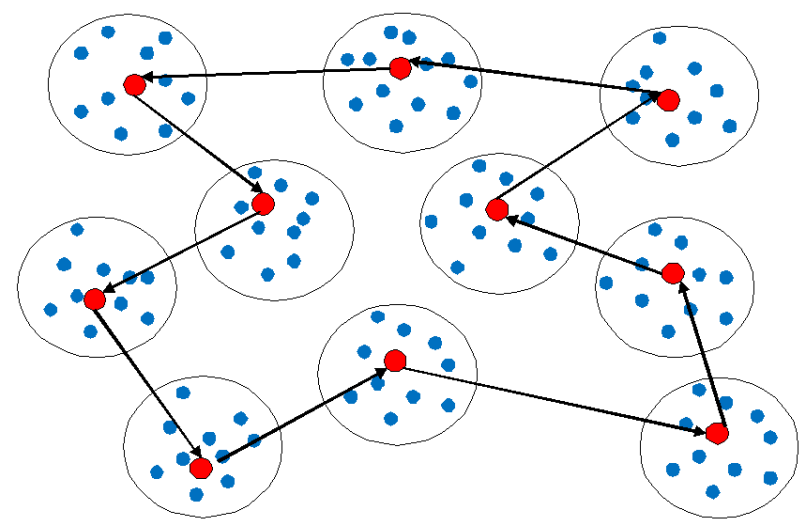

Figure 11. Data gathering from centroid sensor node by mobile base node

The simulated network consists of 10 clusters and it is assumed that clusters are already formed, energy invested to find the trajectory of mobile data gathering node is measured under DGA and CGA over 10 trails. Experiments are carried by considering two different starting points for data gathering node. In the first case the starting point for mobile data gathering node is at first cluster while in the second case the starting point is at seventh cluster. Figure 12 shows the trajectory convergence in CGA and DGA for modile node starting from 1st cluster, and Figure 13 gives convergence for mobile node starting from 7th cluster. The performance of DGA and CGA in terms of invested energy and obtained trajectory for mobile node starting from 1st cluster is shown in Table 5 and starting from 7th cluster in Table 6 respectively, and it is observed that DGA consumes less energy than CGA. Similarly Table 7 and Table 8 shows the performance of DGA and CGA with mobile node starting position from 7th cluster. It has observed that very less energy invested trajectory has been opted by DGA compared to CGA. The performance of mean convergence over 10 independent trials has also shown in Figure 12 and Figure 13 and in both cases a significant improvement has been observed with DGA compared to CGA.

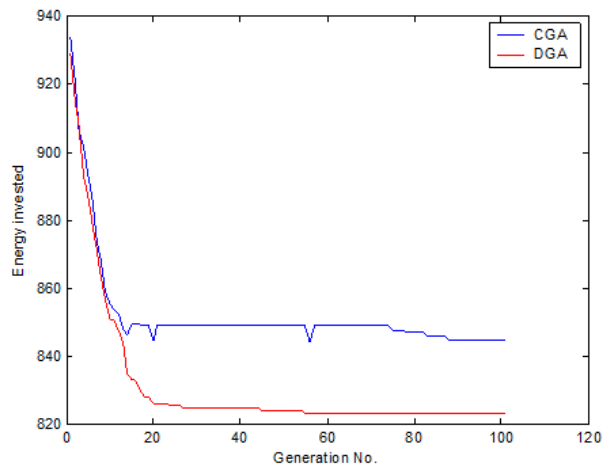

Figure 12. Trajectory Convergence in CGA and DGA over 10 independent trials with starting position in 1 st cluster

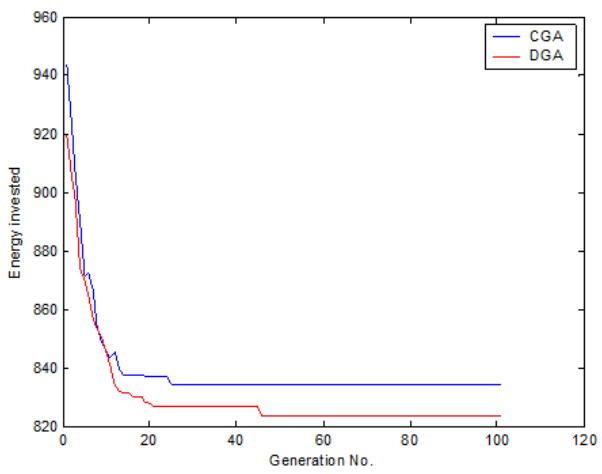

Figure 13. Convergence in CGA and DGA over 10 independent trials with starting position is 7 th cluster 
Table 5. Performance under 10 trials by DGA

\begin{tabular}{|c|c|c|c|c|c|c|c|c|c|c|c|c|}
\hline Trial No. & & & & bta & ed 1 & aje & pry & $\overline{\text { DC }}$ & & & & Invested Energy \\
\hline 1 & 1 & 6 & 7 & 8 & 5 & 3 & 10 & 2 & 9 & 4 & 1 & 818 \\
\hline 2 & 1 & 4 & 9 & 2 & 10 & 3 & 5 & 8 & 7 & 6 & 1 & 818 \\
\hline 3 & 1 & 5 & 3 & 9 & 4 & 2 & 10 & 6 & 7 & 8 & 1 & 836 \\
\hline 4 & 1 & 4 & 9 & 2 & 10 & 3 & 5 & 8 & 7 & 6 & 1 & 818 \\
\hline 5 & 1 & 4 & 9 & 2 & 10 & 3 & 5 & 8 & 7 & 6 & 1 & 818 \\
\hline 6 & 1 & 6 & 7 & 8 & 5 & 3 & 10 & 2 & 9 & 4 & 1 & 818 \\
\hline 7 & 1 & 4 & 9 & 2 & 10 & 3 & 5 & 8 & 7 & 6 & 1 & 818 \\
\hline 8 & 1 & 4 & 9 & 3 & 5 & 7 & 8 & 2 & 10 & 6 & 1 & 825 \\
\hline 9 & 1 & 4 & 9 & 2 & 8 & 7 & 6 & 10 & 3 & 5 & 1 & 826 \\
\hline 10 & 1 & 6 & 10 & 2 & 4 & 9 & 3 & 5 & 7 & 8 & 1 & 836 \\
\hline & & & & & & & & & \multicolumn{3}{|c|}{ Mean } & 823.1 \\
\hline & & & & & & & & & \multicolumn{3}{|c|}{ Std. Dev. } & 7.46 \\
\hline
\end{tabular}

Table 6. Performance under 10 trials by CGA

\begin{tabular}{ccccccccccccc}
\hline Trial No. & \multicolumn{10}{c}{ Obtained Trajectory by CGA } & Invested Energy \\
\hline 1 & 1 & 3 & 5 & 8 & 7 & 6 & 10 & 2 & 9 & 4 & 1 & 832 \\
2 & 1 & 4 & 5 & 3 & 9 & 2 & 10 & 6 & 7 & 8 & 1 & 863 \\
3 & 1 & 2 & 9 & 10 & 6 & 7 & 8 & 5 & 3 & 4 & 1 & 862 \\
4 & 1 & 4 & 2 & 9 & 3 & 5 & 7 & 8 & 10 & 6 & 1 & 855 \\
5 & 1 & 8 & 7 & 5 & 3 & 9 & 4 & 2 & 10 & 6 & 1 & 836 \\
6 & 1 & 4 & 9 & 2 & 10 & 3 & 5 & 8 & 7 & 6 & 1 & 818 \\
7 & 1 & 5 & 3 & 9 & 4 & 2 & 10 & 6 & 7 & 8 & 1 & 836 \\
8 & 1 & 4 & 2 & 9 & 3 & 5 & 7 & 8 & 10 & 6 & 1 & 855 \\
9 & 1 & 4 & 9 & 2 & 10 & 6 & 7 & 8 & 5 & 3 & 1 & 832 \\
10 & 1 & 2 & 8 & 7 & 5 & 3 & 4 & 9 & 10 & 6 & 1 & 860 \\
& & & & & & & & & & & Mean & 844.9 \\
& & & & & & & & & & Std. Dev. & 15.87 \\
\hline
\end{tabular}

Table 7. Performance under 10 trials by CGA

\begin{tabular}{|c|c|c|c|c|c|c|c|c|c|c|c|c|}
\hline Trial No. & & & & bta & & aje & ory & $\mathrm{CC}$ & & & & Invested Energy \\
\hline 1 & 7 & 6 & 1 & 4 & 9 & 2 & 10 & 3 & 5 & 8 & 7 & 818 \\
\hline 2 & 7 & 8 & 2 & 9 & 4 & 1 & 5 & 3 & 10 & 6 & 7 & 826 \\
\hline 3 & 7 & 8 & 2 & 9 & 4 & 1 & 6 & 10 & 3 & 5 & 7 & 826 \\
\hline 4 & 7 & 5 & 3 & 9 & 4 & 1 & 6 & 10 & 2 & 8 & 7 & 825 \\
\hline 5 & 7 & 6 & 10 & 2 & 4 & 9 & 3 & 5 & 1 & 8 & 7 & 836 \\
\hline 6 & 7 & 6 & 1 & 5 & 3 & 10 & 9 & 4 & 2 & 8 & 7 & 846 \\
\hline 7 & 7 & 8 & 2 & 4 & 9 & 10 & 3 & 5 & 1 & 6 & 7 & 846 \\
\hline 8 & 7 & 6 & 10 & 2 & 4 & 9 & 3 & 5 & 1 & 8 & 7 & 836 \\
\hline 9 & 7 & 6 & 10 & 2 & 1 & 4 & 9 & 3 & 5 & 8 & 7 & 830 \\
\hline 10 & 7 & 5 & 3 & 9 & 2 & 4 & 1 & 6 & 10 & 8 & 7 & 855 \\
\hline \multicolumn{12}{|c|}{ Mean } & 834.4 \\
\hline \multicolumn{12}{|c|}{ Std. Dev. } & 11.6 \\
\hline
\end{tabular}

Table 8 . Performance under 10 trials by DGA

\begin{tabular}{|c|c|c|c|c|c|c|c|c|c|c|c|c|}
\hline Trial No. & & & & bta & $\overline{\operatorname{ed~T}}$ & ije & ry 1 & $\overline{\mathrm{DG}}$ & & & & Invested Energy \\
\hline 1 & 7 & 8 & 2 & 10 & 6 & 1 & 4 & 9 & 3 & 5 & 7 & 825 \\
\hline 2 & 7 & 8 & 2 & 9 & 4 & 1 & 5 & 3 & 10 & 6 & 7 & 826 \\
\hline 3 & 7 & 6 & 1 & 4 & 9 & 2 & 10 & 3 & 5 & 8 & 7 & 818 \\
\hline 4 & 7 & 6 & 10 & 3 & 5 & 1 & 4 & 9 & 2 & 8 & 7 & 826 \\
\hline 5 & 7 & 6 & 1 & 4 & 9 & 2 & 10 & 3 & 5 & 8 & 7 & 818 \\
\hline 6 & 7 & 6 & 1 & 4 & 9 & 2 & 10 & 3 & 5 & 8 & 7 & 818 \\
\hline 7 & 7 & 6 & 1 & 4 & 9 & 2 & 10 & 3 & 5 & 8 & 7 & 818 \\
\hline 8 & 7 & 6 & 10 & 3 & 5 & 1 & 4 & 9 & 2 & 8 & 7 & 826 \\
\hline 9 & 7 & 8 & 1 & 6 & 10 & 2 & 4 & 9 & 3 & 5 & 7 & 836 \\
\hline 10 & 7 & 5 & 3 & 9 & 4 & 1 & 6 & 10 & 2 & 8 & 7 & 825 \\
\hline \multicolumn{12}{|c|}{ Mean } & 823.6 \\
\hline \multicolumn{12}{|c|}{ Std. Dev. } & 5.7 \\
\hline
\end{tabular}




\section{CONCLUSION}

Energy efficient routing is one of the best means to increase the life span of WSN. In this paper sensor to sensor communication has achieved to minimize the route cost while an optimization of mobile data gathering trajectory is made to minimize the invested energy. Proposed solution has achieved with a new form of Genetic algorithm which carry the natural phenomena of suppression of weaker by dominant one. The proposed process has delivered a better routing path in less time as well with high level of reliability compared to conventional form of GA. The achieved trajectory for mobi sink has shown energy efficient along with consistency in the performance.

\section{REFERENCES}

[1] Fengyuan Ren, et al., "EBRP: Energy-Balanced Routing Protocol for Data Gathering in Wireless Sensor Networks “, IEEE Transactions on Parallel and Distributed Systems, vol. 22, Issue. 12, pp. 2108-2125, 2011.

[2] Rong Cui ;Zhaowei Qu ; Sixing Yin, ”Energy-efficient routing protocol for energy harvesting wireless sensor network", in 15th IEEE International Conference on Communication Technology, 2013.

[3] M. Bayani, ”An Overall WSN Lifetime Analysis Using Directed Diffusion and Cluster-Based Protocols “, in Iranian journal of electrical and computer engineering, vol.12, NOs. 1 \& 2, 2013.

[4] Esther M.Arkin, et al., "Data transmission and base-station placement for optimizing the lifetime of wireless sensor networks", in Ad Hoc Networks, vol.12, pp. 201-218, January 2014.

[5] Chaonan Wang, Liudong Xing, "Reliability and lifetime modeling of wireless sensor nodes", in Microelectronics Reliability, vol. 54, Issue. 1, pp. 160-166, January 2014.

[6] Bilal AbuBakr, "Extending Lifetime of Wireless Sensor Networks by Management of Spare Nodes", in Procedia Computer Science, vol. 34, pp. 493-498, 2014.

[7] Mariam Akbar, Nadeem Javaid ,'Sink mobility aware energy-efficient network integrated super heterogeneous protocol for WSNs", in EURASIP Journal on Wireless Communications and Networking, 2016.

[8] Huseyin," Maximizing Wireless Sensor Network lifetime by communication/computation energy optimization of non-repudiation security service: Node level versus network level strategies", in Ad Hoc Networks, vol. 37, Part-2, pp. 301-323, February 2016.

[9] Robert M.Curry,"A survey of optimization algorithms for wireless sensor network lifetime maximization", in Computers \& Industrial Engineering, vol. 101, pp. 145-166, November 2016.

[10] K.F. Man, et al., ," Genetic algorithms: concepts and applications [in engineering design] “, in IEEE Transactions on Industrial Electronics, vol. 43, Issue. 5, October 1996.

[11] Holland J.H. Genetic Algorithms and Adaptation. In: Selfridge O.G., Rissland E.L., Arbib M.A. (eds) Adaptive Control of Ill-Defined Systems. NATO Conference Series (II Systems Science), vol. 16, Springer, Boston, MA, 1984.

[12] Trong-Thua Huynh, et al., "Delay constraint energy-efficient routing based on Lagrange relaxation in wireless sensor networks “, in IET Wireless Sensor Systems, vol. 7, Issue. 5, pp. 138-145, 2017.

[13] Yongjun Sun ; Wenxin Dong, et al., ”An Improved Routing Algorithm Based on Ant Colony Optimization in Wireless Sensor Networks “, in IEEE Communications Letters, vol. 21, Issue. 6, pp. 1317-1320, 2017.

[14] Jian Shen ; Anxi Wang, et al., "An Efficient Centroid-Based Routing Protocol for Energy Management in WSN-Assisted IoT", in IEEE Access, vol. 5, pp. 18469-18479, 2017.

[15] Hsiang-Hung Liu ; et al., "On Energy-Efficient Straight-Line Routing Protocol for Wireless Sensor Networks “, in IEEE Systems Journal, vol. 11, Issue. 4, pp. 2374 - 2382, 2017.

[16] Yahya Kord Tamandani,"Computing geometric median to locate the sink node with the aim of extending the lifetime of wireless sensor networks", in Egyptian Informatics Journal, vol. 18, Issue. 1, pp. 21-27, March 2017.

[17] J.Roselin, P.Latha, et al., "Maximizing the wireless sensor networks lifetime through energy efficient connected coverage", in Ad Hoc Networks, vol. 62, pp. 1-10, July 2017.

[18] Mao Yuxing ; et al., "Weak node protection to maximize the lifetime of wireless sensor networks ", in Journal of Systems Engineering and Electronics, vol. 29, Issue. 4, pp. 693-706, 2018.

[19] Turki A. Alghamdi," Secure and Energy Efficient Path Optimization Technique in Wireless Sensor Networks Using DH Method “, in IEEE Access, vol. 6, pp. 53576 - 53582, 2018.

[20] Sandeep Verma, et al., "Design of a novel routing architecture for harsh environment monitoring in het- 
erogeneous WSN “,in IET Wireless Sensor Systems, vol. 8, Issue. 6, 2018.

[21] Jianhua Qiao, et al., "Polar Coordinate-Based Energy-Efficient-Chain Routing in Wireless Sensor Networks Using Random Projection “, in IEEE Access, vol. 6, pp. 21275 - 21286, 2018.

[22] Felicia Engmann,'Prolonging the Lifetime of Wireless Sensor Networks: A Review of Current Techniques", in Wireless Communications and Mobile Computing, 2018.

[23] Mohamed Elshrkawey, et al., "An Enhancement Approach for Reducing the Energy Consumption in Wireless Sensor Networks", in Journal of King Saud University - Computer and Information Sciences, vol. 30, Issue. 2, pp. 259-267, April 2018.

[24] Arun L.Kakhandki, et al., "Energy efficient selective hop selection optimization to maximize lifetime of wireless sensor network", in Alexandria Engineering Journal, vol. 57, Issue. 2, pp. 711-718, June 2018.

[25] Manisha R. Dhage, Srikanth Vemuru," Routing Design Issues in Heterogeneous Wireless Sensor Network", in International Journal of Electrical and Computer Engineering (IJECE), Vol.8, No.2, April2018, pp. $1028-1039$.

[26] Hemavathi P, Nandakumar A N," Novel Bacteria Foraging Optimization for Energy -efficient Communication in Wireless Sensor Network", in International Journal of Electrical and Computer Engineering (IJECE),Vol.8, No.6, December 2018, pp. 4755 - 4762.

[27] Basavaraj G.N, Jaidhar C.D,' Low latency and energy efficient cluster based routing design for wireless sensor network", in Indonesian Journal of Electrical Engineering and Computer Science,Vol.13 , No.2, February2019, pp. 615 - 625.

[28] Chaitra HV, Dr. Ravikumar G.K ," Energy efficient clustering method for wireless sensor network", in Indonesian Journal of Electrical Engineering and Computer Science,Vol.14, No.2, May2019, pp. 1039 1048 .

\section{BIOGRAPHIES OF AUTHORS}

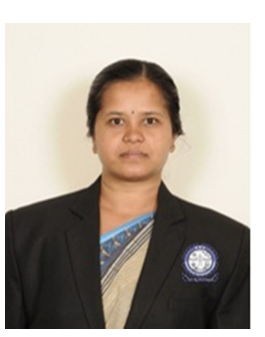

Prof. Shanthi D L is a graduate research scholar at Viswesvaraya Technological University Belagavi Karnataka with Master of Technology from Viswesvaraya Technological University Belagavi India (2007). Currently working as Assistant Professor at BMS Institute of Technology Bangalore India. Her researches are in fields of Wireless Sensor Networks, Networks, Internet of Things. She is affiliated with IEEE as student member. Further info on homepage: https://bmsit.ac.in

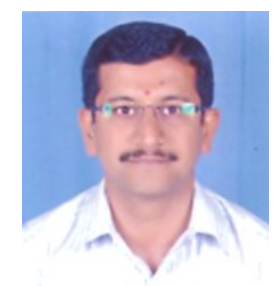

Dr. Keshava Prasanna Professor and Dean (Students) at CIT Tumkur India. is a research guide at Viswesvaraya Technological University Belagavi Karnataka India. He is having vast experience in teaching and guiding research scholars. His researches are in fields of Wireless Sensor Networks, Pattern Recognition, Image Processing, Brain Computer Interfacing etc, . He is a member of IEEE, chair for various conferences, reviewer for various conferences and journals. Further info on homepage: https://www.cittumkur.org 